\title{
How effective are 'age' tools at changing patient behaviour? A rapid review
}

\section{Bavidra Kulendrarajah 도 , Adam Grey, David Nunan}

10.1136/bmjebm-2019-111244

Centre for Evidence-Based Medicine, Oxford University, Oxford, UK

Correspondence to: Dr Bavidra Kulendrarajah, Centre for Evidence-Based Medicine, Oxford University, Oxford OX1 2JD, UK; bavidra10@gmail.com

\section{Abstract}

Background A common form of risk communication is to relay the relative risk (\%) of an adverse outcome based on surrogate markers associated with the outcome. A novel way of communicating risk is through 'effective age' of a person or specific organ. These tools can be used to change patient behaviour.

Objective To determine the effect of 'effective age' tools on patient behaviour as compared with more traditional methods of risk communication.

Study selection We performed a search of the PubMed database up to February 2019 for systematic reviews and randomised controlled trials (RCT) that answered our question. Interventions were 'effective age' tools, comparators were usual care or alternative risk communication tools. Primary outcomes were behavioural change measures.

Findings We included 1 overview of systematic reviews (level 1 evidence), 2 systematic reviews (level 1 evidence) and 13 RCTs (level 2 evidence). Both systematic reviews concluded the evidence base was not conclusive enough to make specific recommendations.

Age tools assessed in the 13 RCTs were: 'lung age' $(n=5)$, 'heart age' $(n=3)$, 'health age' $(n=2)$, 'cardiovascular age' $(n=1)$, 'body age' $(n=1)$ and 'net present value' $(\mathrm{n}=1) .7 / 13$ (54\%) RCTs demonstrated a clinical effect on behaviour change favouring the 'age' tool; 2/13 (15\%) demonstrated a null effect; 4/13 (31\%) favoured control.

Conclusions Our findings indicate that systematic review evidence needs updating. The evidence from RCTs on the effect of using age metrics on patient behaviour is poor. There is a need for high-quality trials to decrease uncertainty in the available evidence.

\section{Background}

Chronic risk associated with an adverse life event can be communicated in many ways. Risk measures are typically calculated as the predicted probability of having an adverse life event during the next 5-10 years. An alternative risk communication tool is through "effective age" ${ }^{1}$ of a person or specific organ. Multiple terms are used to express 'effective age', including: heart age, ${ }^{2}$ lung age, ${ }^{3}$ brain age, ${ }^{4}$ health age, ${ }^{5}$ vitality age $e^{6}$ and biological age. $^{7}$ The idea is that patients compare their 'scientific' age calculated from various risk factors to their chronological age. For example, if the chronological age is 50 but the calculated effective age is 60 , this means that the patient is in the same risk category as the 60 years old who has no risk factors. This allows for a more vivid representation of their risk and ideally should promote positive changes in their behaviour.

The two most commonly used 'age scores' are 'heart age' and 'lung age'. Heart age is the age of someone of the same gender and ethnicity who has healthy risk factors and a matching annual risk of heart attack or stroke. The National Health Service uses the JBS3 risk calculator ${ }^{8}$ to estimate 'heart age'. The model uses multivariable modelling and takes into account risk factors such as blood pressure, smoking, cholesterol and diabetes. Lung age established by Morris and Temple in $1985^{9}$ is calculated based on spirometry readings.

Our aim was to perform a rapid evaluation of the evidence assessing the effect of an 'effective age' compared with relative and absolute measures for communicating adverse health risk on subsequent patient behaviour and health outcomes. Another aim of this brief review was to determine if a more comprehensive systematic review is warranted.

\section{Objectives}

The main objective was to examine the effect of 'effective age' tools for communicating risk and changing patient behaviour. This could be changes in objective clinical measurements such as cholesterol levels or blood pressure or in behaviours such as smoking or exercise levels.

\section{Methods}

\section{Criteria for considering studies}

\section{Types of studies}

Systematic reviews and randomised controlled trials (RCT). We also examined observational studies in the process of the review. However, due to the number of higher level of evidence studies found we made the decision to restrict our review to only systematic reviews and RCTs.

\section{Participants}

Individuals who were over the age of 18 interacting with a health service. There were no restrictions about the type of healthcare service that they were interacting with nor did we exclude studies based on comorbidities of the populations involved.

\section{Interventions}

Any intervention that uses a body or organ 'age' calculation. We describe elsewhere the variety of these calculations. The variety of these tools is broad and as such the criteria for acceptance into 
this review were broad without the need for the calculator to be validated elsewhere.

\section{Comparator}

The comparators needed to avoid the use of an 'age' calculation. Otherwise, we included studies which used other methods of risk communication or centred on the use of 'usual care'.

\section{Outcome measurements}

The main outcome measurements were centred on a behavioural change measure. This may have included cessation from smoking or increases in physical activity. Intention to change behaviour was included in the initial search as were surrogate outcome measures, for example, cholesterol changes. But we restricted this to behavioural change or surrogate outcomes and excluded intention to change behaviour.

\section{Search methods}

We performed searches of the PubMed database using search criteria distilled from background reading. The search terms used initially were 'Lung age' and 'Heart age'. We then hand searched the references of these studies and used the 'related articles' feature of PubMed to find other possible articles for inclusion. We also used these articles to find other potential search terms. The terms arising from this search included 'Fitness age', 'Body age', 'Effective age', 'Brain age', 'Vascular age', 'Risk advancement period', 'Rate advancement period', 'Real age', 'Risk age', 'Health age', 'Age gap', 'Microlife', 'Net present value', 'Biologic age' and 'Biological age'. Finally, we searched Trip and Scholar using the same search terms.

\section{Reasons for exclusion}

Studies were excluded from final analysis if they did not meet our initial participant-intervention-comparator-outcome measurement (PICO). Following data extraction we also excluded studies if their primary outcomes were intention to change behaviour rather than surrogate outcomes for, or actual behaviour change. This was due to the number of studies found using these more robust outcomes.

\section{Evaluation of studies}

We evaluated RCTs using the Cochrane revised risk of bias tool for RCT (RoB 2 tool). ${ }^{10}$ To evaluate systematic reviews we used the AMSTAR 2 tool. $^{11}$

\section{Results}

We identified 1 systematic review of systematic reviews, 2 systematic reviews and 13 RCTs that met the inclusion criteria. This included trials assessing the impact of various 'effective age' measures as defined in table 1.

The systematic review of systematic reviews ${ }^{12}$ included nine systematic reviews, one of which we include in the next section. ${ }^{13}$ This showed little evidence that personalised risk communication had any effect on behaviour, but did not look solely at 'effective age' tools, rather generic personalisation of risk communication. They highlight the level of heterogeneity within all of the studies as well as the overall poor quality of studies included within the reviews as potential issues with drawing meaningful conclusions.

\section{Evidence from systematic reviews}

The two systematic reviews (Bize et $a l^{14}$ and Waldron et $a l^{13}$ ) broadly assessed the effect of different risk communication strategies for smoking cessation and cardiovascular risk, respectively, without focusing specifically on 'age' based tools. However, the systematic reviews were unable to pool their results as the heterogeneity of the studies was too great. Bize et $a l^{14}$ looked at how giving smokers feedback on the physical effects of smoking using physiological measurements (eg, carbon monoxide measurement or lung function tests) affected smoking cessation rates. This Cochrane review scored highly on the AMSTAR 2 tool (11 out of 16). Out of the 15 studies, only one ${ }^{15}$ was relevant to our question. Waldron et $a l^{13}$ compared different interventions used to communicate cardiovascular risk and assess their impact on patient-related outcomes. Like Parkes, this review also concluded that better quality trials were needed to compare the different methods of risk presentation before conclusions can be drawn to which one is most effective. This systematic review also scored poorly on AMSTAR 2 (5/16).

\section{Evidence from RCTs}

The 13 RCTs examined a number of different 'age' metrics. The risk of bias associated with these studies is shown in figure 1; overall few studies had a low risk of bias (3/13), with key issues being poor or poorly described randomisation (9/13) and allocation concealment (11/13) methods.

Data for 18 outcomes are provided in table 1; 7/18 outcomes demonstrated clinical and statistical benefit in favour of the intervention; 3/18 suggested clinical benefit for intervention but did not meet statistical significance; 8/18 showed no clinical difference and were not statistically significant, two of which favoured control (see table 1 and figure 1). figure 2 illustrates the bias associated with the studies.

\section{Cardiovascular age}

Four RCTs looked at the effect of heart age/cardiovascular age on patient behaviour. Bonner et $a l^{16}$ showed that at 2 weeks after intervention, there was a positive change in lifestyle. The control group, who were told absolute cardiovascular risk, had a 15\% smoking cessation rate. The intervention group achieved 23\%. Although the difference was not statistically significant, we feel the results are clinically significant. Lopez-Gonzalez et $a l^{17}$ also showed that informing patients about the heart age resulted in a positive change behaviour. There was a statistically greater reduction in the number of smokers $(-1.8 \%$ vs $0.9 \%)$, greater number of physical activity sessions per week (3.6 vs 2.3) and greater improvement in metabolic parameters at 12 months in the intervention group told their heart age versus the control group who were just given conventional medical advice. Lowensteyn et $a l^{18}$ showed that informing patients their heart age resulted in a greater proportion of high-risk patients returning for a follow-up general practitioner (GP) appointment compared with the control group not told their health age (OR 1.23 vs 0.77 ). Grover et $a l^{19}$ indirectly showed improvement in behaviour in the intervention group through measuring lipid profiles. There was a significantly greater reduction in mean low-density lipoprotein cholesterol levels $(-51 \mathrm{mg} / \mathrm{dL}$ vs $-48.0 \mathrm{mg} / \mathrm{dL})$ and the total cholesterol to high-density lipoprotein cholesterol ratio (1.5 vs 1.3 ) in patients receiving cardiovascular age compared with usual care.

\section{Lung age}

Five RCTs looked at the effect of spirometric-lung-age and associated smoking cessation. Three of these studies (Parkes et $a l^{15}$, Kaminsky et $a l^{20}$ and Takagi et $a l^{21}$ ) showed that the intervention group had a clinically significant increase in smoking cessation. Parkes et $a l^{15}$ showed that at 12 months the intervention group 


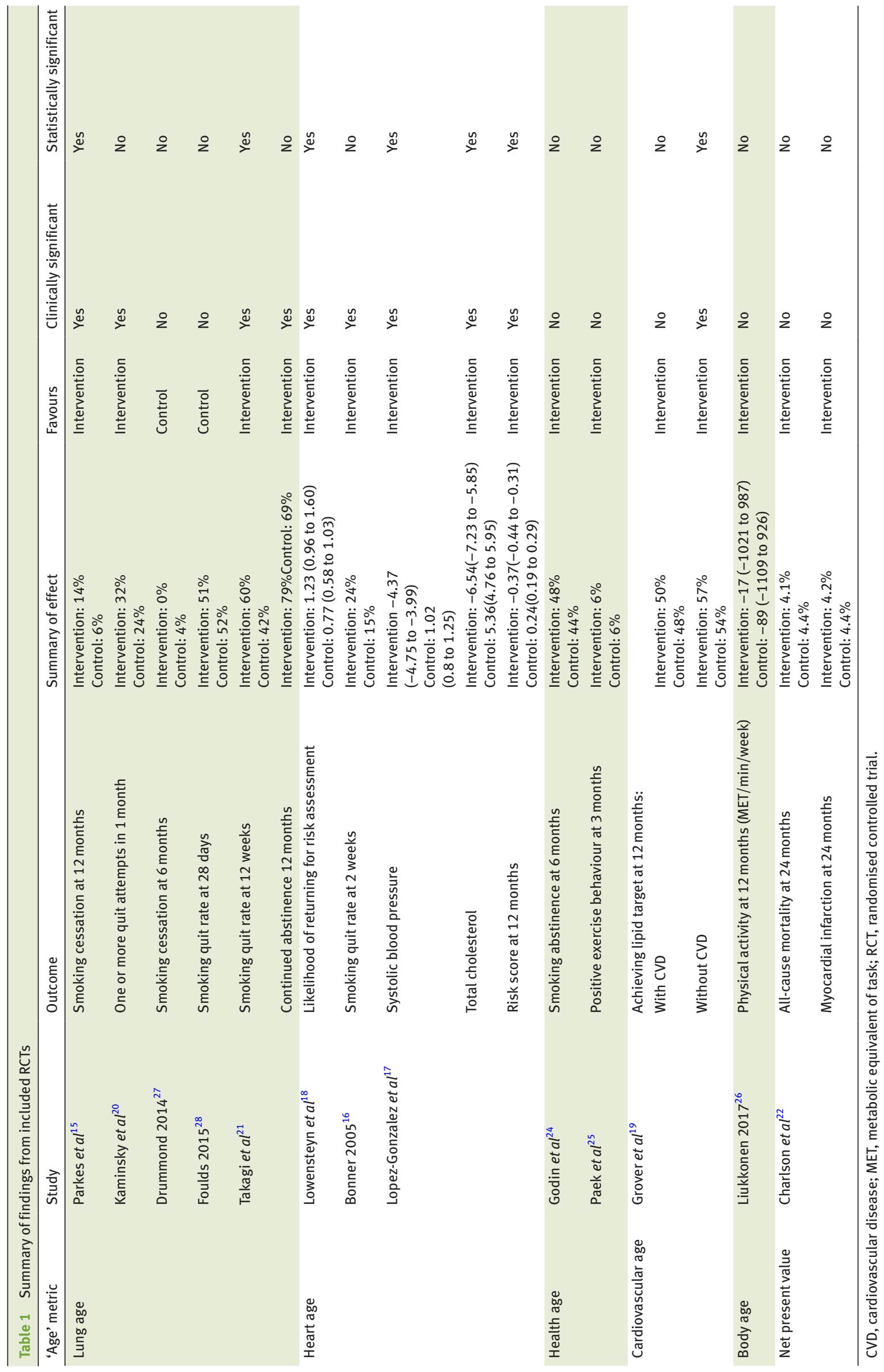

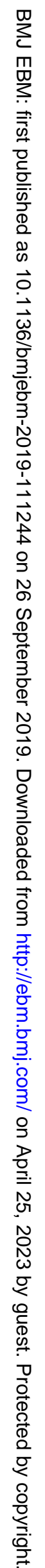




\section{Rapid review results}

Overview of systematic reviews

Systematic reviews

RCT

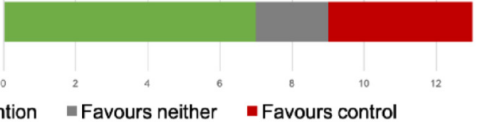

Figure 1 A bar chart summarising clinically significant results. RCT, randomised controlled trial.

had a confirmed smoking cessation rate of $13.6 \%$ versus control at $6.4 \%(\mathrm{p}=0.01)$. There was also significantly lower mean daily cigarette consumption at 12 months in the intervention group compared with the control (11.7 vs $13.7, \mathrm{p}=0.03$ ). Kaminsky et $a l^{20}$ reported quit rates of $32 \%$ in the intervention group versus $24 \%$ in the control group. In the study by Takagi et $a l^{21}$ the smoking quit rate at 12 weeks in the intervention group was 59.6\% vs $41.9 \%$ in the control group. This effect was sustained at the 1-year continued abstinence rate (78.6\% vs 69\%).

\section{Other age tools}

Other RCTs looking at different age metrics including biological age, body age and health age showed no significant effect on behaviour. Charlson et $a l^{22}$ investigated methods of framing risk to patients who had undergone angioplasty. The intervention group were asked to choose a selection of risk factors they wanted to change from a list that showed biological age reduction associated with each risk factor. The control group chose from a list that showed how the risk factors deviated from normal values and were told that changing these would increase life span. At 24 months, change in behaviour was assessed as number free from death, myocardial infarction and stroke. However, there were no significant differences between the two groups.

Liukkoen et $a l^{23}$ reported that informing participants of their body age compared with giving them feedback on their performance alone had no effect on the amount of physical activity

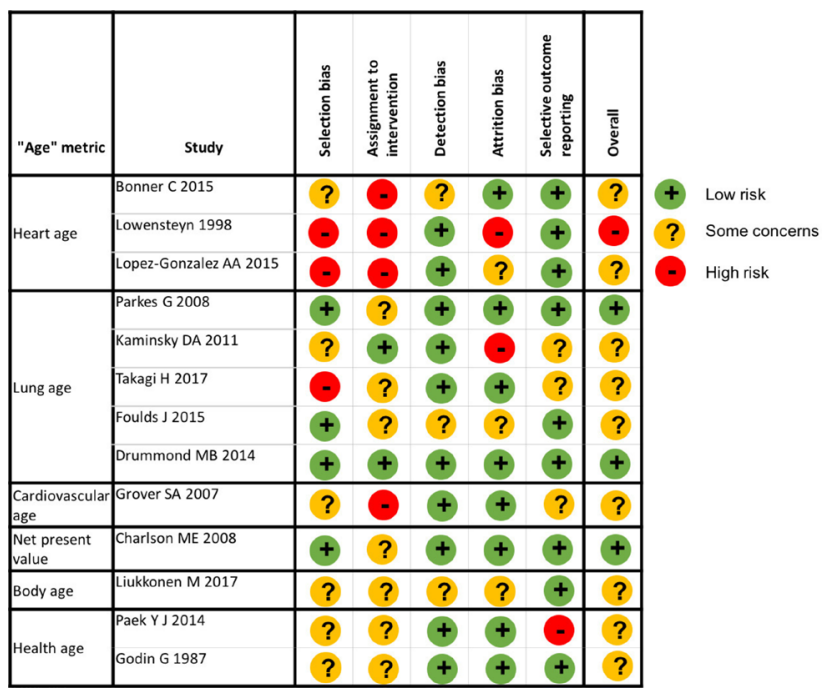

Figure 2 Table showing the risk of bias associated with each study. Bias assessed using the Cochrane revised risk of bias tool (RoB 2 tool). between the two groups. Similarly, Godin et $a l^{24}$ showed no significant change in physical activity at 3 months between groups either given health age or feedback on physical activity.

Paek et $a l^{25}$ showed that informing participants of their health age had no effect on smoking abstinence rates at 6 months. The control group given conventional counselling had a validated smoking abstinence of $17.6 \%$ compared with the intervention group given their health age who had a rate of $21.6 \%$. The difference between the two groups was $4 \%(-4.5 \%$ to $12.5 \%)$.

\section{Discussion}

Our rapid literature search has shown that communication of chronic risk through 'body/organ age' may have a positive impact on patient behaviour. Studies investigating lung age have shown that intervention results in greater smoking cessation rates. Cardiovascular age studies suggest improvement in surrogate outcome measures, including cholesterol and blood pressure, with others suggesting greater intention to eat healthily and do more physical activities. However, the impact of other age metrics, including body, health and biological age, appears to have less of an impact on patient behaviour. This could be related to the validity of the age tools. The best validated tools (and those used in practice) are the 'heart age' and 'lung age' tools. It is perhaps not surprising that there are therefore more studies and that these studies are of better methodological quality.

Meta-analyses of the data were unable to be performed in any of the reviews. This was mostly due to the heterogeneity of the recruitment, measurements of patient behaviour and the use of different age calculators. Recruitment included GP practices, ${ }^{15} 1820$ Foulds $2015{ }^{19}$ college campuses (Lipkus 2007), online access research panels (Souretti 2010, Dammon OC 2018), market research company recruitment, ${ }^{16}$ smoking cessation clinics, ${ }^{21} 25$ hospital records (Drummond 2014, ${ }^{22}$ ) employee from recycling company, ${ }^{26}$ telephone directory ${ }^{24}$ and Spanish public health workers. ${ }^{17}$ Therefore, a meta-analysis could not be performed on the data.

All but three studies were judged to be of high or unclear risk of bias in at least one domain. The key issues being poor or poorly described randomisation (9/13) and allocation concealment $(11 / 13)$ methods. This was echoed by the reviews which highlighted the poor methodological quality of many of the studies included. This is highly likely to affect the conclusions of any systematic review performed presently. Another limitation we found was the difference in intervention between groups within studies. Intervention groups often had more motivation and explanation of the benefits of behaviour change when compared with the control groups. This may have influenced the findings in some of the studies.

The reviews included in this literature search were completed over 6 years ago (with the exception of the overview which does not include many of the trials included here). As such our search encountered many new trials which were not available at the time. With the inclusion of the newly published studies we believe there may be enough homogeneity of studies, particularly within the lung and cardiovascular age tools for a further systematic review to take pace. This could update the previous reviews and further them with the inclusion of a meta-analysis. Issues of poor trial methodology will, however, still remain. We also recommend further, methodologically robust, trials of 'effective age' tools for risk communication.

Twitter David Nunan@dnunan79 
Contributors BK and AG both contributed equally to data collection and write-up of this manuscript. DN supervised the data collection.

Funding The authors have not declared a specific grant for this research from any funding agency in the public, commercial or not-for-profit sectors.

\section{Competing interests None declared.}

Patient consent for publication Not required.

Provenance and peer review Not commissioned; externally peer reviewed.

Data availability statement All data relevant to the study are included in the article or uploaded as supplementary information.

\section{ORCID iDs}

Bavidra Kulendrarajah http://orcid.org/0000-0002-0624-7800

David Nunan http://orcid.org/0000-0003-4597-1276

\section{References}

1. Spiegelhalter D. How old are you, really? Communicating chronic risk through 'effective age' of your body and organs. BMC Med Inform Decis Mak 2016;16:104.

2. NHS Health Check - NHS Choices. Heart age calculator [Internet]. Available: https://www.nhs.uk/conditions/nhs-health-check/pages/checkyour-heart-age-tool.aspx [Accessed 3 Dec 2015].

3. Lung Age For Smoking Cessation [Internet].. Available: http://www.chestxray.com/index.php/calculators/lung-age-for-smoking-cessation [Accessed cited 2015 Dec 3].

4. Mail Online. Try test that reveals your brain age and read how to knock years off [Internet]. Mail Online.. Available: http://www.dailymail.co.uk/ health/article-2821157/Try-test-reveals-brain-age-read-knock-years-it.html [Accessed cited 2015 Dec 3].

5. PreventDisease.com. Health Age and Life Expectancy [Internet]. [. Available: http://preventdisease.com/healthtools/articles/health_age.shtml [Accessed cited 2015 Dec 3].

6. Vitality Age. Vitality Age - Your details [Internet]. Available: https://www. vitalityage.co.uk/ [Accessed 3 Dec 2015].

7. BBC Guides. Take the test: What's my real age?. [online], 2019. Available: http://www.bbc.co.uk/guides/zg3hk7h [Accessed 20 Jun. 2019].

8. Joint British Societies' consensus recommendations for the prevention of cardiovascular disease (JBS3). Heart 2014;100(Suppl 2):ii1-67.

9. Morris JF, Temple W. Spirometric "lung age" estimation for motivating smoking cessation. Prev Med 1985;14:655-62.

10. Higgins JPT, Sterne JAC, Savović J, et al. A revised tool for assessing risk of bias in randomized trials. In: Chandler J, McKenzie J, Boutron I, Welch $V$ (editors). Cochrane methods. Cochrane database of systematic reviews. 10, 2016.

11. Shea BJ, Reeves BC, Wells G, et al. AMSTAR 2: a critical appraisal tool for systematic reviews that include randomised or non-randomised studies of healthcare interventions, or both. BMJ 2017;358.
12. French DP, Cameron E, Benton JS, et al. Can communicating personalised disease risk promote healthy behaviour change? A systematic review of systematic reviews. Ann Behav Med 2017;51:718-29.

13. Waldron C-A, van der Weijden T, Ludt S, et al. What are effective strategies to communicate cardiovascular risk information to patients? A systematic review. Patient Educ Couns 2011;82:169-81.

14. Bize R, Burnard B, Mueller Y, et al. Biomedial risk assessment as an aid for smoking cessation. Cochrane database of systematic reviews 2012;12.

15. Parkes G, Greenhalgh T, Griffin M, et al. Effect on smoking quit rate of telling patients their lung age: the Step2quit randomised controlled trial. BMJ 2008;336:598-600.

16. Bonner C, Jansen J, Newell BR, et al. Is the "Heart Age" Concept Helpful or Harmful Compared to Absolute Cardiovascular Disease Risk? An Experimental Study. Med Decis Making 2015;35:967-78.

17. Lopez-Gonzalez AA, Aguilo A, Frontera M, et al. Effectiveness of the heart age tool for improving modifiable cardiovascular risk factors in a southern European population: a randomized trial. Eur J Prev Cardiol 2015;22:389-96.

18. Lowensteyn I, Joseph L, Levinton C, et al. Can computerized risk profiles help patients improve their coronary risk? the results of the coronary health assessment study (CHAS). Prev Med 1998;27:730-7.

19. Grover SAet al. Patient knowledge of coronary risk profile improves the effectiveness of dyslipidemia therapy. Arch Intern Med 2007;167:2296.

20. Kaminsky DA, Marcy T, Dorwaldt A, et al. Motivating smokers in the hospital pulmonary function laboratory to quit smoking by use of the lung age concept. Nicotine Tob Res 2011;13:1161-6.

21. Takagi H, Morio Y, Ishiwata T, et al. Effect of telling patients their "spirometric-lung-age" on smoking cessation in Japanese smokers. $J$ Thorac Dis 2017;9:5052-60.

22. Charlson ME, Peterson JC, Boutin Foster C, et al. Changing health behaviours to improve health outcomes after angioplasty: a randomised trial to net present value versus future value risk communication. Health education research 2008.

23. Liukkoen M, Nygard C-H, Laukkeun R. A cluster randomised control trial on the effects of technology aided testing and feedback on physical activity and biological age among employees in a medium sized enterprise. Safety and Health at work 2017.

24. Godin G, Desharnias R, Jobin J, et al. The impact of physical fitness and health age appraisal upon exercise intentions and behaviour. Journal of Behavioural Mediicne 1987;10.

25. Paek Y-J, Lee S, Kim Y-H, et al. Effect on smoking quit rate of telling smokers their health risk appraisal in terms of health age: a randomized control trial. Asian Pac J Cancer Prev 2014;15:4963-8.

26. Liukkonen M, Nygård C-H, Laukkanen R. A cluster randomized controlled trial on the effects of Technology-aided testing and feedback on physical activity and biological age among employees in a medium-sized enterprise. Saf Health Work 2017;8:393-7.

27. Drummond MB, Astemborski J, Lambert AA, et al. A randomized study of contingency management and spirometric lung age for motivating smoking cessation among injection drug users. BMC Public Health 2014;14:761.

28. Foulds J, Veldheer S, Hrabovsky S, et al. The effect of motivational lung age feedback on short-term quit rates in smokers seeking intensive group treatment: a randomized controlled pilot study. Drug Alcohol Depend 2015;153:271-7. 